\title{
Explanation of Nikiforov-Uvarov method in quantum mechanics - with a view toward algebraic geometry
}

\author{
Ichio Kikuchi ${ }^{1}$, Akihito Kikuchi ${ }^{2} *$ \\ ${ }^{1}$ Internationales Forschungszentrum für Quantentechnik \\ ${ }^{2}$ International Research Center for Quantum Engineering, Tokyo
}

March 1,2020

\begin{abstract}
In this essay, we review the Nikiforov-Uvarov method which is used to solve Schrödinger equation. We shed light on the algorithm from the viewpoint of algebraic geometry so that we shall the ideas of the latter (such as the resolution of singularity, normalization, primary decomposition of ideal) are lurking behind the algorithm. Besides, we study the application of the introductory D-module theory in this sort of eigenvalue problem and we present an algorithm alternative to the Nikiforov-Uvarov method.
\end{abstract}

\section{Introduction}

In [1, the authors (I. K. and A. K.) have pointed out the relation between algebraic geometry and quantum mechanics. It is summarized as follows. In quantum mechanics (with Heisenberg picture), the basic equation is given by the secular equation of matrix; the eigenvalue problem is, from the viewpoint of algebraic geometry, the primary ideal decomposition; the primary decomposition is related to the basic ideas of algebraic geometry, such as the normalization and the resolution of singularities. This circumstance betokens some sort of relation between the Schrödinger equation in quantum mechanics and algebraic geometry since the matrix representation is derived from the basis set expansion of the wave-function. In the first part of this article, we shall see that this foresight is actually valid; indeed, the example of this is the Nikiforov-Uvarov algorithm (which is a procedure to obtain analytical solutions of Schrödinger equation). In recent years, the interest for this method seems to be resurrected, and we can find several articles on this topic, although these references are given in technical and pragmatic manners to solve the equation [2, 3, 4, 5]. In contrast to them, in the first part of this article, we shall peep into the latent ideas of algebraic geometry which works inside the algorithm. In the second part, we sketch the theory of D-modules as a useful tool for the problems of quantum mechanics. As a small application of the D-module theory, we present an algorithm alternative to the Nikiforov-Uvarov method.

\footnotetext{
*akihito_kikuchi@gakushikai.jp (The corresponding author)
} 


\section{Part I}

\section{Nikiforov-Uvarov Method from the viewpoint of algebraic geometry}

\section{The Nikiforov-Uvarov Method}

Let us review the Nikiforov-Uvarov Method, which enables us to analytically solve the timeindependent Scrödinger equation. At first, we should manage to transform the Schrödinger equation into the following form:

$$
\psi^{\prime \prime}(s)+\frac{\tilde{\tau}(s)}{\sigma(s)} \psi^{\prime}(s)+\frac{\tilde{\sigma}(s)}{\sigma(s)} \psi^{\prime}(s)=0,
$$

where $\sigma(s)$ and $\tilde{\sigma}(s)$ are the polynomials of at most second-degree; $\tilde{\tau}(s)$ is of the first-degree; thus $\psi(s)$ is the function of the hypergeometric type.

If we assume $\psi(s)=\phi(s) y(s)$, we obtain

$$
y^{\prime \prime}(s)+\left(\frac{2 \phi^{\prime}(s)}{\phi(s)}+\frac{\tilde{\tau}(s)}{\sigma(s)}\right) y^{\prime}(s)+\left(\frac{\phi^{\prime \prime}(s)}{\phi(s)}+\frac{\phi^{\prime}(s)}{\phi(s)} \frac{\tilde{\tau}(s)}{\sigma(s)}+\frac{\tilde{\sigma}(s)}{\sigma^{2}(s)}\right) y(s)=0 .
$$

Let us rewrite the second coefficients in the left hand side of Eq. 2 as follows:

$$
\frac{\tau(s)}{\sigma(s)}:=\left(\frac{\phi^{\prime}(s)}{\phi(s)}+\frac{\tilde{\tau}(s)}{\sigma(s)}\right)
$$

with a new polynomial $\tau(s)$. In this definition, we rewrite the term in the right hand side as

$$
\frac{\pi(s)}{\sigma(s)}:=\frac{\phi^{\prime}(s)}{\phi(s)}
$$

with a new polynomial

$$
\pi(s)=\frac{1}{2}[\tau(s)-\tilde{\tau}(s)] .
$$

Here $\pi(s)$ is the polynomial of at most one degree.

From these definitions, we obtain the relation:

$$
\frac{\phi^{\prime \prime}(s)}{\phi(s)}=\left(\frac{\phi^{\prime}(s)}{\phi(s)}\right)^{\prime}+\left(\frac{\phi^{\prime}(s)}{\phi(s)}\right)^{2}=\left(\frac{\pi(s)}{\sigma(s)}\right)^{\prime}+\left(\frac{\pi(s)}{\phi(s)}\right)^{2} .
$$

Hence we can rewrite the coefficient of the third term in the left hand side of Eq.2 by

$$
\frac{\bar{\sigma}(s)}{\sigma^{2}(s)}:=\frac{\phi^{\prime \prime}(s)}{\phi(s)}+\frac{\phi^{\prime}(s)}{\phi(s)} \frac{\tilde{\tau}(s)}{\sigma(s)}+\frac{\tilde{\sigma}(s)}{\sigma^{2}(s)},
$$

where

$$
\bar{\sigma}(s)=\tilde{\sigma}(s)+\pi^{2}(s)+\pi(s)\left[\tilde{\tau}(s)-\sigma^{\prime}(s)\right]+\pi^{\prime}(s) \sigma(s)
$$

Then we arrive at the equation:

$$
y^{\prime \prime}(s)+\frac{\tau(s)}{\sigma(s)} y^{\prime}(s)+\frac{\bar{\sigma}(s)}{\sigma^{2}(s)} y(s)=0 .
$$

If we could use a new particularization

$$
\bar{\sigma}(s)=\lambda \sigma(s)
$$


with an indeterminate $\lambda$, we would rewrite the equation as

$$
\sigma(s) y^{\prime \prime}(s)+\tau(s) y^{\prime}(s)+\lambda y(s)=0 .
$$

Here we require that $\lambda$ should be independent of $s$ in order to solve the eigenvalue problem; then the equation is of the hypergeometric type.

Because of Eq. (8) and Eq. 10, the first order polynomial $\pi(s)$ should satisfy the quadratic equation

$$
\pi^{2}(s)+\pi(s)\left[\tilde{\tau}(s)-\sigma^{\prime}(s)\right]+\tilde{\sigma}(s)-k \sigma(s)=0,
$$

where

$$
k=\lambda-\pi^{\prime}(s)
$$

As $\pi(s)$ is of the first degree with respect to $s$, the variable $k$ is independent of $s$. In other words, we compute $\pi(s)$ as the solutions to the quadratic equation; and we would get a polynomial relation between $\lambda$ and with the constants, such as the energy $E$ and the others, involved in the given differential equation. We defer the demonstration of how to compute $\lambda$, as it is highly case-dependent.

At present, $y(s)$ and $\lambda$ are still unknowns in the sense of eigenvalue problem. According to the theory of hypergeometric function [6], the relevant solutions to Eq. 111)(indexed by integers $n=0,1,2, \ldots)$ are given by

$$
y_{n}(s)=\frac{B_{n}}{\rho(s)} \frac{d^{n}}{d s^{n}}\left[\sigma^{n}(s) \rho(s)\right]
$$

where $B_{n}$ is the normalization constant, and $\rho(s)$ is the function which should satisfy the relation,

$$
(\sigma(s) \rho(s))^{\prime}=\tau(s) \rho(s)
$$

or

And $\lambda_{n}$ is given by

$$
\frac{1}{\rho(s)} \frac{d \rho(s)}{d s}=\frac{\tau(s)-\sigma^{\prime}(s)}{\sigma(s)}
$$

$$
\lambda_{n}=-n \tau^{\prime}(s)-\frac{n(n-1)}{2} \sigma^{\prime \prime}(s) .
$$

Now we should remember that we have prepared the analytic expression of $\lambda$ by solving the quadratic equation for $\pi(s)$. If we equate this analytic representation of $\lambda$ (from the quadratic equation) and $\lambda_{n}$, this will be an equation for the eigenvalues $E=E_{n}$, because $\lambda$ would be a function of $E$.

Remark. In order to get general eigenvalue solutions $y_{n}(s)$ of Eq. 11, we should construct a suitable polynomial $\left\{\tau_{n}(s)\right\}$ and a suitable $\left\{\lambda_{n}\right\}$ for each $y_{n}(s)$. (Recall that $\tau(s)$ and $\pi(s)$ are adjustable polynomials for the purpose of eigenvalue solution.) To this end, let us define a new function $w_{n}(s)$ by

$$
w_{n}(s):=\rho(s) y_{n}(s) .
$$

The weight function $\rho(s)$ by Eq. 15 is chosen in such a way that the differential equation [Eq. [11]] could be transformed into the following form:

$$
\frac{d}{d s}\left[\rho(s) \sigma(s) y_{n}^{\prime}(s)\right]+\lambda_{n} \rho(s) y_{n}(s)=0 .
$$

Provisionally, let us abandon the definition of $\lambda_{n}$ in Eq.17) and let us assume it to be an independent variable, associated to $y_{n}(s)$. Then we rewrite Eq. 19. as

$$
\sigma(s) w_{n}^{\prime \prime}(s)+\left[2 \sigma^{\prime}(s)-\tau(s)\right] w_{n}^{\prime}(s)+\left[\sigma^{\prime \prime}(s)-\tau^{\prime}(s)+\lambda_{n}\right] w_{n}(s)=0 .
$$


Let us define those functions:

$$
\begin{gathered}
r_{n}(s):=(2-n) \sigma^{\prime}(s)-\tau(s), \\
\mu_{n}:=(1-n) \sigma^{\prime \prime}(s)-\tau^{\prime}(s),
\end{gathered}
$$

and

$$
\varepsilon_{n}:=-\frac{n(n-1)}{2} \sigma^{\prime \prime}(s)-n \tau^{\prime}(s) .
$$

Let us introduce a subsidiary function $v_{n}(s)$, and let us define a function $P(s)$ by

$$
P(s):=\sigma(s) v_{n}^{\prime \prime}(s)+r_{n}(s) v_{n}^{\prime}(s)+\mu_{n} v_{n}(s) .
$$

If $P(s)$ is a polynomial of degree $n-1$, we have the identity:

$$
\begin{aligned}
0=\frac{d^{n} P(s)}{d s^{n}} & =\frac{d^{n}}{d s^{n}}\left[\sigma(s) v_{n}^{\prime \prime}(s)+r_{n}(s) v_{n}^{\prime}(s)+\mu_{n} v_{n}(s)\right] \\
& =\sigma(s) z_{n}^{\prime \prime}(s)+\left[2 \sigma^{\prime}(s)-\tau(s)\right] z_{n}^{\prime}(s)+\left[\sigma^{\prime \prime}(s)-\tau^{\prime}(s)+\varepsilon_{n}\right] z_{n}(s),
\end{aligned}
$$

with

$$
z_{n}(z):=v_{n}^{(n)}(z)
$$

Note that the right hand side of Eq. 25 is equivalent to the left hand side of Eq. 20). Then we could choose $z_{n}(s)$ as the solution $w_{n}(s)$ of the latter equation and we could set $\lambda_{n}=\varepsilon_{n}$. Moreover, let us assume that we could choose $v_{n}(s)$ such that $P(s)=0$. Concerning the right hand side of Eq.24, we should have

$$
0=\sigma(s) v_{n}^{\prime \prime}(s)+\tau_{n}(s) v_{n}^{\prime}(s)+\mu_{n} v_{n}(s)=\frac{d}{d s}\left[\sigma(s) v_{n}^{\prime}(s)+\left((1-n) \sigma^{\prime}(s)-\tau(s)\right) v_{n}(s)\right] .
$$

By means of the weight function $\rho(s)$, we actually find a suitable representation of $v_{n}(s)$ as

$$
v_{n}(s)=B_{n} \sigma^{n}(s) \rho(s)
$$

which satisfies the requirement from Eq. (27). It leads to the representation of $y_{n}(s)$ given in Eq. 14.

\section{Resolution of Singularities}

We make a detour in this section for the sake of discussions henceforward. Let us inspect the plane curve:

$$
y^{2}=x^{3}+x^{2}
$$

It is converted into two equations:

$$
y= \pm x \sqrt{1+x}
$$

As we see in the curve depicted in the figure 2, Eq. 30 represents the two branches of the plane curve at the origin, at which a singularity (a node) lies.

Let us do the following transformation:

$$
x=u, y=u v
$$

Then we obtain

$$
u^{2}\left(v^{2}-u-1\right)=0
$$

This equation represents two duplicated lines $u=0$ and a parabola $v^{2}-u-1=0$. This transformation is called "blowing up". Neither the lines nor the parabola contains the node at the origin. In this sense, we have done the "resolution of singularities". Note that the 


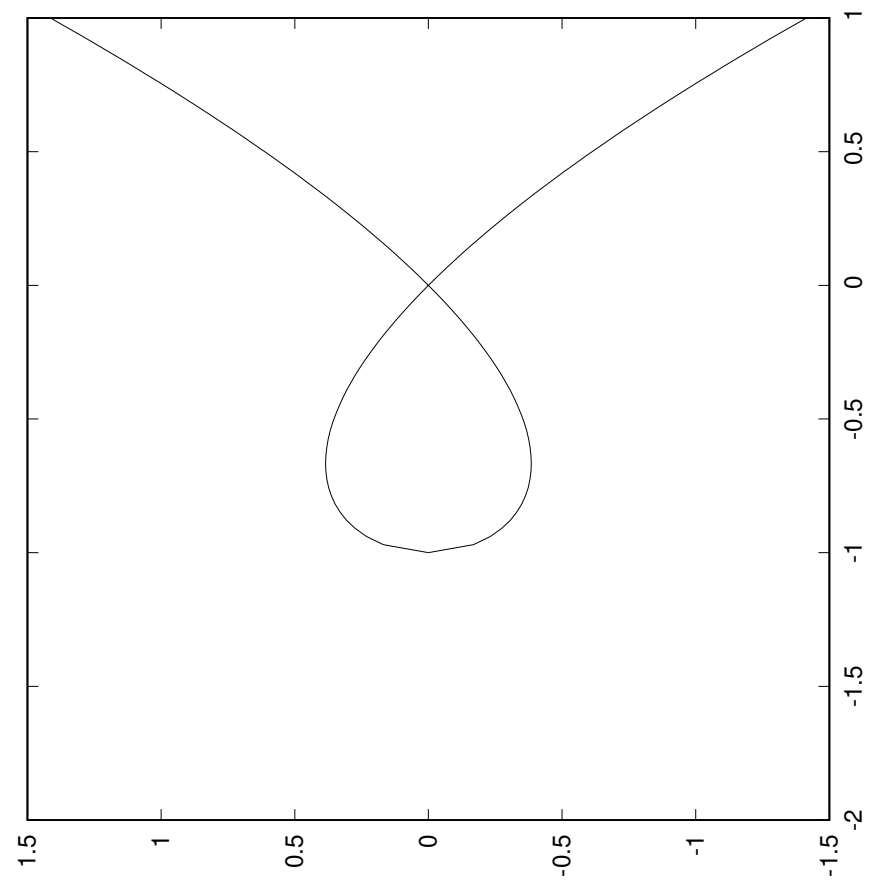

Figure 1: The image of a plane curve $y^{2}=x^{3}+x^{2}$. 
blowing up is done with the change of variables, through which the curve is mapped into another coordinate system.

The resolution of singularity is related to the idea of the "normalization"; and the "normalization" of algebraic variety (a geometrical object depicted by the set of polynomial equations) is related to the "primary ideal decomposition". These three computational processes are conducted by quite different algorithms, but the latter two are often useful for the same purpose as the first, to remove singularities in algebraic variety.

In order to comprehend the idea of "primary ideal decomposition", let us consider the two-level model in quantum mechanics:

$$
\left(\begin{array}{cc}
0 & -1 \\
-1 & 0
\end{array}\right)\left(\begin{array}{l}
x \\
y
\end{array}\right)=e\left(\begin{array}{l}
x \\
y
\end{array}\right)
$$

This is the set of polynomial equations: $x+e y=y+e x=0$. The polynomials define an ideal $I$ in $\mathbb{R}[x, y, e]$, and the ideal is decomposed into primary ideals:

$$
I=\left(x+e y, y+e x, x^{2}+y^{2}-1\right)=(e-1, y e+x) \cap(e+1, y e+x) \cap(x, y)
$$

If we add the normalization condition of the solution vector $\left(x^{2}+y^{2}-1=0\right)$ to the equations, the new ideal is decomposed as

$$
J=\left(x+e y, y+e x, x^{2}+y^{2}-1\right)=\left(e-1,2 y^{2}-1, y e+x\right) \cap\left(e-1,2 y^{2}-1, y e+x\right)
$$

Observe that the decomposition reveals the solutions of the polynomial equations when we compute the common zero of the simplified polynomials in the decomposed ideals therein. Indeed, such a simplification of the polynomial equations is the common feature of the primary ideal decomposition and the normalization of the algebraic variety. Note that the decomposition of the ideal is the decomposition of the eigenspace; namely, the primary ideal decomposition serves as the solution of the eigenvalue problem (which quantum mechanics always requires in the Heisenberg picture). As for the details of this sort of argument, see 1].

\section{Examination of Nikiforov-Uvarov algorithm from the viewpoint of algebraic geometry}

In this section, we turn back to quantum mechanics. Let us consider the radial Schrödinger equation for hydrogen-like atom:

$$
\frac{d^{2} R(s)}{d s^{2}}+\frac{2}{s} \frac{d R(s)}{d s}+\frac{2 \mu}{\hbar^{2}}\left[E+\frac{Z e^{2}}{s}-\frac{\hbar^{2} l(l+1)}{2 \mu s^{2}}\right] R(s)=0 .
$$

The eigenvalue and the angular quantum number are denoted by $E$ and $l$. In the atomic unit (in which $\mu=1, e=1$ and $\hbar=1$ ), the equation is rewritten as

$$
\frac{d^{2} R(s)}{d s^{2}}+\frac{2}{s} \frac{d R(s)}{d s}+\frac{1}{s^{2}}\left[-\alpha^{2} s^{2}+\beta s-l(l+1)\right] R(s)=0,
$$

where

$$
\alpha^{2}=-2 E, \beta=2 Z .
$$

For the convenience of algebra, we use $\alpha^{2}$.

The polynomials required by the Nikiforov-Uvarov algorithm are given by

$$
\tilde{\tau}=2, \sigma(s)=s, \tilde{\sigma}=-\alpha^{2} s^{2}+\beta s-l(l+1)
$$

And for $\pi(s)$ (being still unknown), there is a relation:

$$
\bar{\sigma}(s)=\tilde{\sigma}(s)+\pi^{2}(s)+\pi(s)\left[\tilde{\tau}(s)-\sigma^{\prime}(s)\right]+\pi^{\prime}(s) \sigma(s)=-\alpha^{2} s^{2}+\beta s-l(l+1)+\pi^{2}(s)+\pi(s)+s \pi^{\prime}(s) .
$$


In this case, directly we have obtained the equation to which the Nikiforov-Uvarov algorithm is applied. However, this is not the case in general situations; often we have to find a suitable change of the coordinate $r=r(s)$ before we try the algorithm.

We chose the local parameter $\lambda$ such that

$$
\bar{\sigma}(s)=\lambda \sigma(s)=\lambda(s) s .
$$

Here we should notice the analogy to the algorithm of resolution of singularities (that of blowing up). In the latter, the pair of variables $(x, y)$ is transformed to $(u, u v)$ for the resolution of singularities. As is remarked in the previous section, the eigenvalue problem (in matrix theory) could be comprehensible in the context of the ideas concerning the resolution of singularities. In the Nikiforov-Uvarov algorithm, this kind of simple blowing-up is the corner-stone to computational steps, on which the idea of algebraic geometry plays an active part.

The aforesaid linear polynomial $\pi(s)$ should be the polynomial solutions to the equation:

$$
-\alpha^{2} s^{2}+\beta s-l(l+1)+\pi^{2}(s)+\pi(s)+\left(\pi^{\prime}(s)-\lambda\right) s=0
$$

Let us assume $\pi=A s+B$. By the substitution to this equation, we obtain the polynomial relations of $A$ and $B$ as the zero condition of the coefficients:

$$
A^{2}-\alpha^{2}=2 A B+2 A+\beta-\lambda=B^{2}+B-l(l+1)=0 .
$$

In the references [2, 3, 4], $\pi(s)$ is likewise determined as the solution of the quadratic equation $\pi(s)^{2}+f(s) \pi(s)+g(s)=0$ with polynomial coefficients $f(s)$ and $g(s)$ [Eq. 12] ]. The traditional step is to write the solution as

$$
\pi(s)=\frac{-f(s) \pm \sqrt{f(s)^{2}-4 g(s)}}{2}
$$

The solution contains the discriminant $\sqrt{f(s)^{2}-4 g(s)}$; and due to the requirement for polynomial $\pi(s)$, the discriminant must be a square of a polynomial, from which we get the relations among the variables in the given differential equation, as well as the polynomial solutions of $\pi(s)$.

In this article, however, we solve the equation using an ideal-theoretic way (from the standpoint of algebraic geometry). The corresponding ideal of the polynomial equation is given as

$$
I=\left(A^{2}-\alpha^{2}, 2 A B+2 A+\beta-\lambda, B^{2}+B-l(l+1)\right)
$$

Let us execute the primary ideal decomposition of the ideal, using a computer algebra system Singular [7.

The program-script is written as follows:

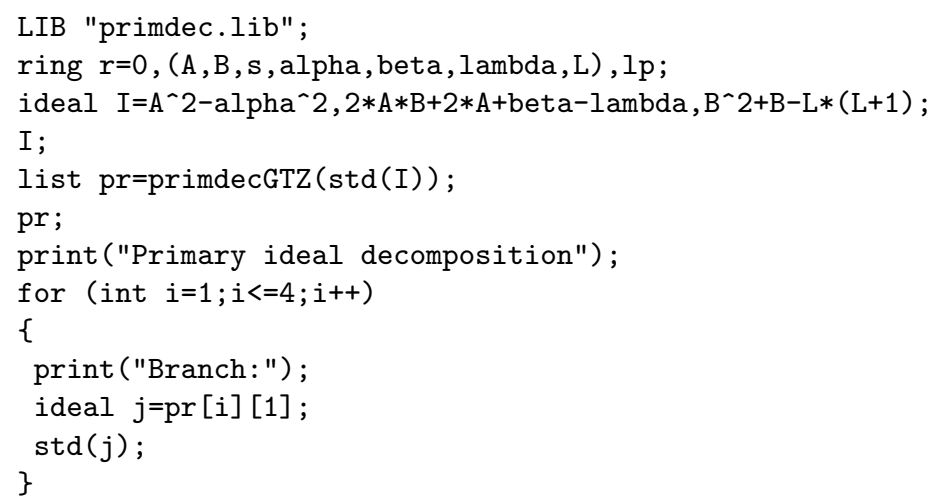

We obtain the result in the following list. 
Primary ideal decomposition

[1] :

[1]: !Primary ideal

_ $[1]=2 *$ alpha $*$ L-beta + lambda

- $[2]=\mathrm{B}+\mathrm{L}+1$

_[3] =A-alpha

[2]: !Corresponding prime ideal;

_ [1] $=2 *$ alpha $*$ L-beta + lambda

[2] $=\mathrm{B}+\mathrm{L}+1$

[3] $=$ A-alpha

[2] :

[1] :

_[1] $=2 *$ alpha $*$ L+beta-lambda

_ $[2]=\mathrm{B}+\mathrm{L}+1$

[2] :

- $[3]=A+a l p h a$

_[1] $=2 *$ alpha $*$ L +beta -1 ambda

_[2] $=\mathrm{B}+\mathrm{L}+1$

[3] $=\mathrm{A}+\mathrm{alpha}$

[3] :

[1] :

_ $[1]=2 * a l$ pha $* \mathrm{~L}+2 *$ alpha - beta + lambda

_ $[2]=B-L$

_ [3] =A+alpha

[2] :

_[1] $=2 *$ alpha $* \mathrm{~L}+2 *$ alpha - beta + lambda

$-[2]=\mathrm{B}-\mathrm{L}$

[3] $=$ A + alpha

[4] :

[1] :

_ [1] $=2 *$ alpha $*$ L $+2 *$ alpha+beta-lambda

- $[2]=B-L$

_[3] =A-alpha

[2] :

_ [1] $=2 *$ alpha $* \mathrm{~L}+2 *$ alpha + beta - lambda

$-[2]=\mathrm{B}-\mathrm{L}$

[3] $=$ A-alpha

In the above list, the primary ideals and the corresponding prime ideals are given respectively. We obtain four primary ideals $p_{i}$ (indexed by [1]...[4]) as the result of the decomposition $\left(I=\cap_{i=1}^{4} p_{i}\right)$ :

$$
\begin{gathered}
p_{1}=(2 \alpha l-\beta+\lambda, B+l+1, A-\alpha), \\
p_{2}=(2 \alpha l+\beta-\lambda, B+l+1, A+\alpha), \\
p_{3}=(2 \alpha l+2 \alpha-\beta+\lambda, B-l, A+\alpha), \\
p_{4}=(2 \alpha l+2 \alpha+\beta-\lambda, B-l, A-\alpha) .
\end{gathered}
$$

Hence there are four possible branches of $\pi$, as is listed in Table 1

Only one branch is admissible as a physically valid solution, owing to the boundary condition $R(0)=R(\infty)=0$. To find admissible one, let us compute the solution of the given differential equation. We choose the third branch and do the integration

$$
\frac{\phi^{\prime}(s)}{\phi(s)}=\frac{\pi(s)}{\sigma(s)}=\frac{-\alpha s+l}{s}
$$




\begin{tabular}{rr}
\hline$\pi(s)$ & $\lambda$ \\
\hline \hline$\alpha s-(l+1)$ & $-2 l \alpha+\beta$ \\
$-\alpha s-(l+1)$ & $2 l \alpha+\beta$ \\
$-\alpha s+l$ & $-2(l+1) \alpha+\beta$ \\
$\alpha s+l$ & $2(l+1) \alpha-\beta$ \\
\hline
\end{tabular}

Table 1: Four possible solutions of $\pi(s)$ and the values of the corresponding $\lambda$.

and obtain

$$
\phi(s)=s^{l} \exp (-\alpha s) .
$$

This solution satisfies the requirement; the other three branches not. We write the solutions of the Schrödinger equation $\psi_{n}(s)=\psi(s) y_{n}(s)$. The eigenvalue to $\psi_{n}(s)$ is computed from

$$
\lambda_{n}=2 n \alpha
$$

and

$$
\lambda=-2(l+1) \alpha+\beta
$$

We then have

$$
\alpha=\frac{\beta}{2+2 n+2 l}
$$

As $\alpha^{2}=-2 E$ and $\beta=2 Z$, the energy $E$ is given by

$$
E_{n}=-\frac{Z^{2}}{2(1+n+l)^{2}}
$$

The relevant functions $y_{n}(s)$ are constructed from the weight function

$$
\frac{d \rho(s)}{d s} \frac{1}{\rho(s)}=\frac{\tau(s)-\sigma^{\prime}(s)}{\sigma(s)}=\frac{1+2 l-2 \alpha s}{s},
$$

namely,

$$
\rho(s)=s^{2 l+1} \exp (-2 \alpha s)
$$

Then we have

$$
y_{n}(s)=B_{n} \frac{1}{\rho(s)} \frac{d^{n}}{d s^{n}}\left(\rho(s) \sigma^{n}(s)\right)=B_{n} \exp (2 \alpha s) s^{-2 l-1} \frac{d^{n}}{d s^{n}}\left(s^{n+2 l+1} \exp (-2 \alpha s)\right) .
$$

Consequently, we obtain

$$
\psi_{n}(s)=\phi(s) y_{n}(s)=N_{n l} s^{l} e^{-a s} y_{n}(s) L_{n}^{2 l+1}(2 \alpha s),
$$

since $y_{n}(s)$ is proportional to the associated Laguerre polynomial $L_{n}^{2 l+1}(2 \alpha s)$

\section{Discussion}

From the argument in the preceding sections, one might see that algebraic geometry is a potential ingredient even in elementary quantum mechanics in the Schrödinger picture. The exemplification of this is the Nikiforov-Uvarov algorithm; the algebraic geometry furnishes the method with the algorithm of "primary ideal decomposition", which determines the possible "branches" of the solutions. We again note here that, in the Heisenberg picture, the inevitable matrix eigenvalue problem could be regarded as a kind of resolution of the 
singularities of the affine algebraic variety and it could be solved by "primary ideal decomposition".

We summarize the procedure in the Nikiforov-Uvarov algorithm as follows.

(1) One has to do "blowing up" [Eq.(10)] as the preparatory step to finding the eigenvalue of the Schrödinger equation. This step is similar to that of the simplest way in algebraic geometry. However, in the Nikiforov-Uvarov algorithm, the "blowing up" induces henceforth more complex computational steps because one knows no singularity explicitly in the beginning; one should seek the location of the singularity through the computation.

(2) After "blowing up", namely, after the local change of the coordinate, one has to find linear polynomial solutions to an equation [Eq. $[12]$ that describes the relation of variables involved therein. The solving process is effectively conducted by the ideal theory of multivariate polynomials, and computational algebraic geometry offers the algorithm, namely, the "primary ideal decomposition", from which four possible branches of the linear polynomial are derived. (The physically admissible branch is elicited from the boundary condition.)

Now we conclude that the eigenvalue problem in quantum mechanics (both of Heisenberg and Schrödinger pictures) is an application of algebraic geometry.

In connection with differential equations, the theory of D-modules is a strong tool to handle them; moreover, this theory borrows and refines a lot of motifs from algebraic geometry. At present, the theory of D-modules is equipped with strong computational algorithms; thanks to them, one can orderly derive the solution of the set of differential equations; with the aid of computer algebra, one can decompose the initial large problem into small ones, which are more tamed and more tractable. Hence the theory of D-modules seems to have the potential to be a powerful tool in quantum mechanics, although, as far as the authors aware, there is no general effective algorithm for the eigenvalue problem in it. Then what should be effective algorithms? Consider the differential operator of the one-dimensional harmonic oscillator,

$$
J=\partial_{x}^{2}+\left(E-x^{2}\right) .
$$

When $E=1$, we can decompose it as

$$
J=\left(\partial_{x}-x\right)\left(\partial_{x}+x\right)
$$

Then, thanks to the simplicity of the problem, we find a solution by

$$
\left(\partial_{x}+x\right) f_{0}(x)=0
$$

and we write the other solutions by

$$
f_{n}(x)=\left(\partial_{x}-x\right)^{n} f_{0}(x) .
$$

We would like to hold this sort of algorithm to " divide and conquer" the problems into the set of smaller ones, in which we shall find the tangible clue. Indeed, the theory D-modules encompasses algorithms for the decomposition of differential operators [8, 9]. Especially, the algorithm given in 9 might be useful in quantum mechanics, although that method is not intended for the search of eigenvalues. (Concerning this, in the second part of the present article, we give a small account of the theory of D-modules and present a symbolic algorithm to solve the eigenvalue problem, flavored by the idea of D-modules) However, it seems that, for quantitative computations in quantum mechanics, the availability of the theory of Dmodule is not ascertained as yet, since the symbolic operation of operators, even if it is employed to the processing of toy models, amounts to a vast quantity of computations, for which the efficacy of the latest architectures of computers might be powerless. It is a more serious matter that the theory of D-modules is built for the study of the linear differential operators, although quantum physics in the real universe is featured by the non-linearity due to the mandatory many-body interactions. Toward this "material" difficulty, the practical expedient is the principle of quantum chemistry: it is a method of approximation by a finite set of basis functions with the radical reduction of the degrees of freedom; traditionally 
the numerical problems encountered therein have been solved by numerical linear algebra. In contrast, if one intends to advance with the prospect of symbolic computation, one should translate the problem into the language of commutative algebraic geometry, as is demonstrated in [10. We have to wait for, or we have to develop by ourselves, more powerful algorithms of computational algebraic geometry applicable in quantum mechanics.

\section{Computer algebra script}

This script is written for GAP, to compute $\pi(s)$ in Eq. 12). The example problem is that of the case of Hulten's potential, as is explained in 2. At first we define $\tau(s), \sigma(s)$, and $\tilde{\sigma}(s)$ and compute the quadratic polynomial for $\pi(s)$ (which is given by "tobezero"). We construct the ideal (to be decomposed) from the coefficients of the quadratic polynomial. Then we apply the primary decomposition to obtain four branches.

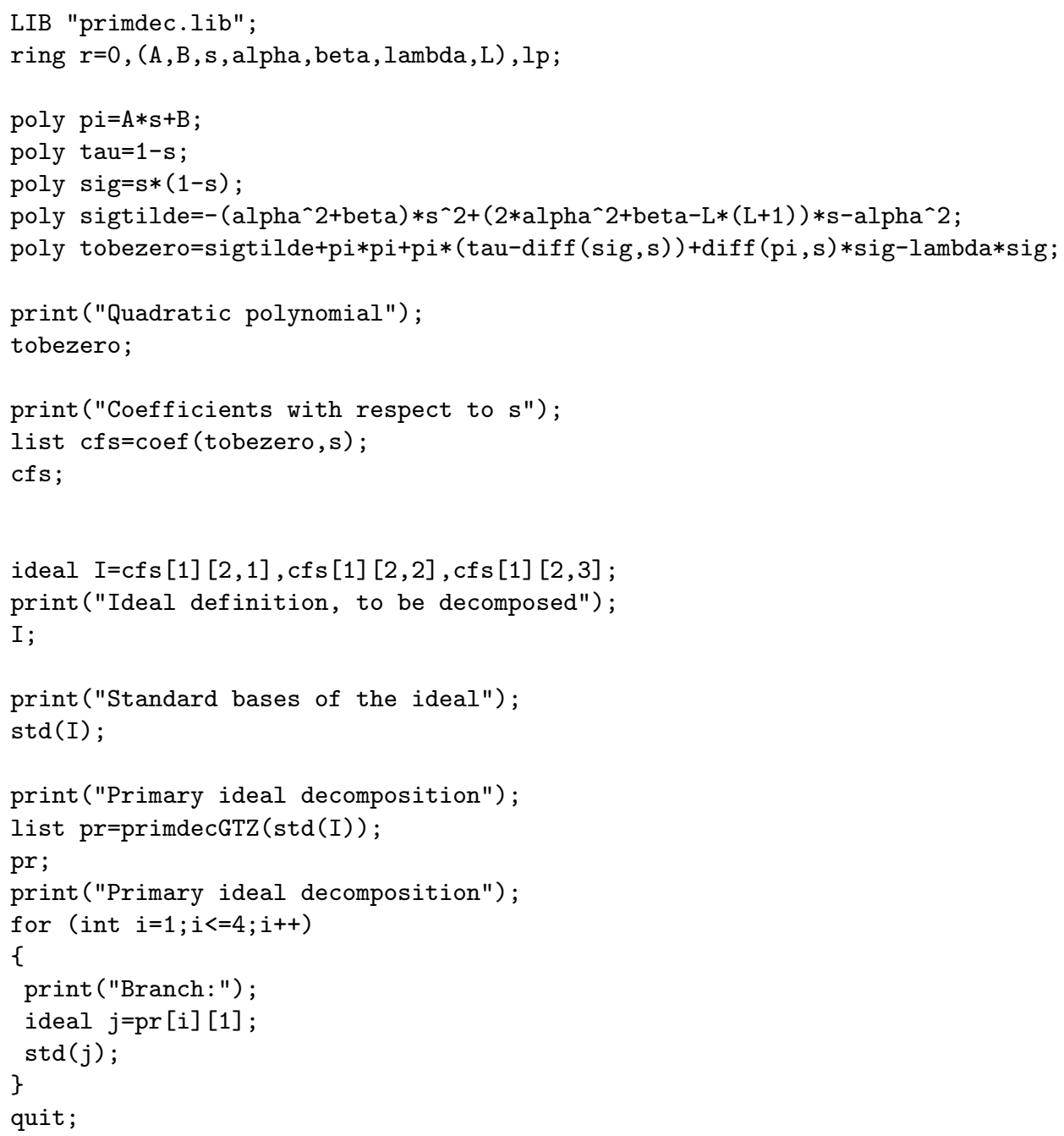




\section{Part II}

\section{A small account of the theory of D-modules and an alternative approach to Nikiforov-Uvarov method}

\section{The theory of D-modules and the application to quantum mechanics}

In this section, we review the several important results of the theory of D-modules so that we would understand how they work in the solution of the Scrödinger equation.

D-modules are defined to be modules over a ring D of differential operators. One uses this mathematical idea to study the solutions of linear partial differential equations with constant coefficients. (For a concise but pragmatical introduction, see 11.) As the theory of D-module is the entrance to a fertile field of mathematics (Algebraic Analysis), one might learn the formal theory from substantial textbooks, such as [12].

We use Weyl algebra with complex coefficients.

$$
D=\mathbb{C}\left[x_{1}, \ldots, x_{n}\right]\left\langle\partial_{1}, \ldots, \partial_{n}\right\rangle,
$$

We abbreviate the basis of the module by

$$
x^{a} \partial^{b}=x_{1}^{a_{1}} x_{2}^{a_{2}} \cdots x_{n}^{a_{n}} \partial_{1}^{b_{1}} \partial_{2}^{b_{2}} \cdots \partial_{n}^{b_{n}} .
$$

Any monomial in D-module can be arranged and written by the normal form of monomials defined as above, through the relation $\left[\partial_{i}, x_{j}\right]=\delta_{i j}$. Hence every operator is given by

$$
P=\sum_{(a, b) \in E} C_{a b} x^{a} \partial^{b}
$$

where $C_{a b}$ is complex number and $E$ is a finite set in $\mathbb{N}^{2 n}$. We need several definitions to set up the weight for each monomial. Let us use two vectors : $u, v \in \mathbb{R}^{n}$ with $u+v \geq 0$. And let us define an integer $m$ as $m=\max _{(a, b) \in E}(a \cdot u+b \cdot v)$.

Definition 1. The initial form of $P$ is defined as

$$
\operatorname{in}_{(u, v)}(P)=\sum_{a \cdot u+b \cdot v=m} C_{a b} \prod_{u_{k}+v_{k}>0} x^{a_{k}} \xi^{b_{k}} \prod_{u_{k}+v_{k}=0} x^{a_{k}} \partial^{b_{k}}
$$

The differential operators form the ideal which we call "D-ideal".

Let $e=(1,1, \ldots, 1)$ and zero $=(0,0, \ldots, 0)$. For a D-ideal $I$, the characteristic ideal is defined as

$$
\operatorname{ch}(I):=\operatorname{in}_{(z e r o, e)}(I) \in \mathbb{C}^{2 n}=\mathbb{C}\left[x_{1}, \ldots, x_{n}, \xi_{1}, \ldots \xi_{n}\right] .
$$

Then the characteristic variety $\operatorname{Char}(I)$ is defined to be the vanishing set of $\operatorname{ch}(I)$. (The vanishing set is the assembly of points on which the value of every polynomial in $\operatorname{ch}(I)$ becomes zero, denoted by $V(\operatorname{ch}(I))$.) By the term "dimension of Char $(I)$ " we refer to the dimension of ideal $I$, i.e. $\operatorname{dim}(I)$. 
Remark. This is an essential question: how to compute the dimension of an ideal?

Let $I$ be an ideal in $\mathbb{C}\left[x_{1}, \ldots, x_{n}\right]$. The dimension of the ideal $(d=\operatorname{dim}(I))$ be the maximum cardinality of the subsets of variables $\left(u \subset\left\{x_{1}, \ldots, x_{n}\right\}\right)$ such that

$$
I \cap \mathbb{C}[u]=(0) .
$$

The following is the statement of "Fundamental theorem of Algebraic Analysis".

Theorem 1 (Theorem 1.10 in [1]). For a proper D-ideal I, every irreducible component of the characteristic variety $\operatorname{Char}(I)$ has dimension at least $n$.

Definition 2. A D-ideal is "holonomic" when $\operatorname{dim}(\operatorname{ch}(I))=n$. Namely, the characteristic variety (in $\mathbb{C}^{2 n}$ ) takes the minimal dimension.

Definition 3. The holonomic rank of a D-ideal $I$ is given by

$$
\operatorname{rank}(I)=\operatorname{dim}_{\mathbb{C}(x)}(\mathbb{C}(x)[\xi] / \mathbb{C}(x)[\xi] \operatorname{ch}(I))
$$

with abbreviations as $x=\left(x_{1}, \ldots, x_{n}\right)$ and $\xi=\left[\xi_{1}, \ldots, \xi_{n}\right]$.

Be careful about the definition: $\mathbb{C}(\mathbf{x})$ is not the ring but the "field" in which polynomials of $x_{1}, \ldots x_{n}$ are invertible; it is the field of rational functions of $x_{1}, \ldots x_{n}$. As $\operatorname{rank}(I)$ is defined in the commutative ring, we could write $\operatorname{rank}(I)$ simply by $R / R I$. If a D-ideal $I$ is holonomic, then $\operatorname{rank}(I)$ is finite. Or we can say that $R I$ is a zero-dimensional ideal. ( An ideal is zero-dimensional if the corresponding affine algebraic variety $V(I)$ is a finite set.)

Definition 4. The singular locus of $I$ is defined as

$$
\operatorname{Sing}(I)=\left(\operatorname{ch}(I):\left\langle\xi_{1}, \ldots, \xi_{n}\right\rangle\right) \cap \mathbb{C}\left[x_{1}, \ldots, x_{n}\right]
$$

through the saturation of ideals $\left(A: B^{\infty}\right)$ with $B^{\infty}=B+B^{2}+\ldots$ and the intersection of ideals $C \cap D$. Namely, it is the closure of the projection of $\operatorname{Char}(I) \backslash \mathbb{C}^{n} \times\{0\}$ onto $\mathbb{C}\left[x_{1}, \ldots, x_{n}\right]$.

Theorem 2 (Cauchy-Kowalevskii-Kashiwara theorem (Theorem 1.14 in [1]). Let I be a holonomic D-ideal; let $U$ be an open, simply connected subset of $\mathbb{C}^{n} \backslash \operatorname{Sing}(I)$. Then the space of holomorphic functions on $U$ (which are the solutions to I) has dimension rank $(I)$. (This theorem holds true only when $\operatorname{rank}(I)$ is finite.)

Let us stand upon these ideas and reconsider a problem in quantum mechanics: the equation for the harmonic oscillator. It is given by

$$
\left(-\frac{1}{2} \frac{d^{2}}{d x^{2}}+x^{2}\right) \psi(x)=E \psi(x)
$$

with the lowest eigenvalue $E=1 / 2$. The corresponding D-ideal is given by

$$
I=\left(\partial_{x}^{2}+\left(1-x^{2}\right)\right) .
$$

Then

and

$$
\operatorname{ch}(I)=\xi^{2}
$$

$$
\operatorname{rank}(I)=2
$$

According to the remark concerning the dimension of an ideal, $\operatorname{ch}(I)$ is of dimension 1 . Hence $I$ is holonomic. From Cauchy-Kowalevskii-Kashiwara theorem, the space of the solutions has dimension 2 (which is equal to $\operatorname{rank}(I)$ ); namely, there are two independent solutions.

Let us see analyze other equations in physics. 
- As an example of $n=2$, the D-ideal for the wave equation is given by

$$
I=\left(\partial_{t}^{2}-\partial_{x}^{2}\right) \in \mathbb{C}[x, t]\left\langle\partial_{x}, \partial_{t}\right\rangle
$$

The characteristic ideal is $c h(I)=\left(\xi_{t}^{2}-\xi_{x}^{2}\right)$ and it has dimension 3 and $\operatorname{rank}(I)$ is infinitesimal. (The latter statement is owing to the reason that $R / R I$ is generated by $\left.1, \xi_{x}, \xi_{x}^{2}, \ldots, \xi_{t} \xi_{x}, \xi_{t} \xi_{x}^{2}, \ldots\right)$ Hence $I$ is not holonomic. We cannot apply CauchyKowalevskii-Kashiwara theorem. Indeed the innumerable solutions of the equation are given by $F(x+t)+G(x-t)$ with $F, G \in C^{2}$.

- From the similar reasoning, the D-ideal for time-dependent Schrödinger equation $i \partial_{t}+\partial_{x}^{2}$ is not holonomic.

- In case of multi-dimensional time-independent Schrödinhger equations:

$$
I=\left(-\frac{1}{2} \sum_{i=1}^{n} \partial_{x_{i}}^{2}+V\left(x_{1}, \ldots, x_{n}\right)-E\right)
$$

with a multivariate polynomial potential $V\left(x_{1}, \ldots, x_{n}\right)$, the characteristic ideal is given by

$$
\operatorname{ch}(I)=\sum_{i} x_{i}^{2}
$$

Then $\operatorname{ch}(I)$ is of dimension $2 n-1$ and $\operatorname{rank}(I)$ is infinite. As $I$ is not holomonic, Cauchy-Kowalevskii-Kashiwara does not hold. This suggests that the space of the solutions would not be limited in dimension $n$.

\subsection{How to solve Schrödinger equation from the viewpoint of the theory of D-modules?}

Let us consider the radial Schrödinger equation for hydrogen-like atom:

$$
\frac{d^{2} R(s)}{d s^{2}}+\frac{2}{s} \frac{d R(s)}{d s}+\frac{1}{s^{2}}\left[-\alpha s^{2}+\beta s-l(l+1)\right] R(s)=0,
$$

Here we employ the differential equation almost identical to that used in the preceding part of this article; the only difference lies in the third term in the left side: $\left[-\alpha s^{2}+\beta s-l(l+1)\right]$; viz. we use $\alpha$ instead of its square. These variables are related to the energy $E$ and the nuclear charge $Z$ by $\alpha=-2 E$ and $\beta=2 Z$.

We rewrite the equation by means of $\theta:=s \partial_{s}$ on behalf of $\partial_{s}$ :

$$
\left(\theta^{2}+\theta+s^{2}\left[2 E+\frac{2}{s}-\frac{l(l+1)}{s^{2}}\right]\right) \phi(S)=0 .
$$

The corresponding D-ideal is given by

$$
J=\left(\theta^{2}+\theta+\left[2 s^{2} E+2 s-l(l+1)\right]\right) .
$$

Let us consider the initial part of $J$ with respect to the weight $(-w, w)$ with $w=1$. It is given by

$$
\operatorname{ind}_{(-w, w)}(J)=\left(\theta^{2}+\theta-l(l+1)\right)=(\theta-l)(\theta+(l+1))
$$

We refer to this ideal by the term "indical ideal", from which we can construct another differential equation; and the solution of this new equation represents the approximate form of the solution of the initially given equation [Theorem 1.22 in [11]]: when the solution of the new equation is given by $x^{u}$ (the power of $x$ ), then the solution of the originally given equation is represented as

$$
x^{u} \cdot g(\log (x))
$$

with a certain function $g$. 
It is noteworthy that we apply the idea of the indical ideal for the eigenvalue problem of quantum mechanics in the framework of hypergeometric ordinary differential equations. The algorithm (which is explained in the following) takes a path different from that of the traditional Nikiforov-Uvarov algorithm, incorporating a kind of the resolution of the singularities.

The indical ideal is decomposed as

$$
J=(\theta-l) \cap(\theta+(l+1))
$$

The former component of the ideal decomposition corresponds to the differential equation:

$$
s \frac{d y}{d s}=l y,
$$

with the solution $y=s^{l}$. This function depicts the behavior of the solution of the radial Schrödinger equation around $s=0$. Hence we could assume a solution with the form of $\phi(s)=s^{l} f(s)$ and we get a new differential equation:

$$
s^{2} f^{\prime \prime}(s)+(2 l+2) f^{\prime}(s)+\left[-\alpha^{2} s^{2}+\beta s\right] f=0 .
$$

We rewrite it by

$$
\left(\theta^{2}+(2 l+1) \theta+\left[-\alpha s^{2}+\beta s\right]\right) f=0 .
$$

Let us decompose the operator $P:=\left(\theta^{2}+(2 l+1) \theta+\left[-\alpha s^{2}+\beta\right]\right)$ as

$$
P=(\theta+W s+X)(\theta Y s+Z)
$$

with the unknowns $W, X, Y, Z$. Notice that the term $\left[-\alpha s^{2}+\beta s\right]$ contains no constant term. As the form of the differential operator suggests, it is natural that we assume a simpler decomposition:

$$
P=(\theta+A s+B)(\theta-A s)=\theta^{2}+B \theta-A s^{2}-A(B+1) s .
$$

By the comparison of the coefficients, we found following equations:

$$
A^{2}=\alpha, B=2 l+1, A(B+1)=-\beta
$$

Namely we obtain

$$
A= \pm \sqrt{\alpha}, B=(2 l+1), \beta=-(2 l+2) \sqrt{\alpha} .
$$

From these relations, we compute $f(s)$ as the solution of the differential equation $(\theta-$ $A s) f(s)=0$, and we find $f(s)=e^{ \pm \sqrt{\alpha} s}$. We construct the admissible solution for the lowest eigenenergy: $\phi(s)=s^{l} e^{-\alpha s}$.

We write the set of solutions of the Schrödinger equation by $\psi_{n}(s)=\phi(s) y_{n}(s)$. Then $y_{n}(s)$ should satisfy Eq. 111), and we can compute related polynomials $(\pi(s)$ and $\tau(s))$ explicitly from $\phi(s)$. To construct relevant solutions $y_{n}(s)$, we use the formula given in Eq. (14). To this end, we employ the framework of the Nikiforov-Uvarov algorithm. We compute $\pi(s)$ by

$$
\frac{\pi(s)}{\sigma(s)}=\frac{\phi^{\prime}(s)}{\phi(s)}=\frac{l}{s}-\sqrt{\alpha}
$$

Since $\sigma(s)=s$, we have

$$
\pi(s)=l-\sqrt{\alpha} \cdot s
$$

And we obtain

$$
\tau(s)=\tilde{\tau}(s)+2 \pi(s)=2 l+2-2 \sqrt{\alpha} s .
$$

Then the quadratic equation for $\pi(s)$ [Eq. 12 ] is given by

$$
\pi(s)^{2}+\pi(s)+\left[-\alpha s^{2}+\beta s-l(l+1)\right]-\left(\lambda-\pi^{\prime}(s)\right) \sigma=0,
$$


which is simplified to

$$
(-2(l+1) \sqrt{\alpha}+\beta-\lambda) s=0
$$

Hence there should be a relation:

$$
(-2(l+1) \sqrt{\alpha}+\beta-\lambda)=0 .
$$

We require that $\lambda$ is equal to $\lambda_{n}$, the latter of which is defined by

$$
\lambda_{n}=-n \frac{d \tau(s)}{d s}-\frac{n(n-1) \sigma^{\prime \prime}(s)}{2}=2 n \sqrt{\alpha} .
$$

As $\alpha$ is proportional to the energy by $E=-\alpha / 2$, we obtain $E_{n}$ from the equation $\lambda=\lambda_{n}$ :

$$
E_{n}=-\frac{Z^{2}}{2(1+n+l)^{2}} \cdot x
$$

We can compute the weight function $\rho(s)$ explicitly from $\phi(s)$ and $\sigma(s)$. Then we obtain the relevant solutions $y_{n}$ of Eq. 14, and also we obtain the solutions of the Schrödinger equation $\psi_{n}(s)=\phi(s) y_{n}(s)$, which are given in the preceding part of this article.

Let us compute another example:

$$
s^{2}(1-s)^{2} \frac{d^{2} \psi(s)}{d s^{2}}+s(1-s)^{2} \frac{d \psi(s)}{d s}+\left[-(\alpha+\beta) s^{2}+(2 \alpha+\beta-l(l+1)) s-\alpha\right] \psi(s)=0
$$

This equation is derived as an approximation of Schrödinger equation with Hulten potential (Equation (167) in [2]); $\beta$ and $l$ are the constants; $\alpha$ is proportional to the energy $E$; the coordinate $s$ is related to the radial $r$ by $\exp (-r / \kappa)$ with a constant $\kappa$.

For this equation, we have

$$
\begin{aligned}
& \sigma(s)=s(1-s) \\
& \tilde{\tau}(s)=1-s \\
& \tilde{\sigma}(s)=-(\alpha+\beta) s^{2}+(2 \alpha+\beta-l(l+1)) s-\alpha .
\end{aligned}
$$

The indical ideal is given by

$$
J_{s}=\left(\theta_{s}^{2}-\alpha\right)=(\theta+\sqrt{\alpha})(\theta-\sqrt{\alpha}) .
$$

Hence the admissible solution would behave like $s^{\sqrt{\alpha}}$ around $s=0$.

As the coefficients in the differential equation include the term $(1-s)$, it would be instructive to infer the form of solution around $s=1$ likewise. We make the change of variables $t=1-s$ and obtain

$$
t^{2}(1-t)^{2} \frac{d^{2} \psi(t)}{d t^{2}}-t^{2}(1-t) \frac{d \psi(t)}{d t}+\left[-(\alpha+\beta) t^{2}+(\beta+l(l+1)) t-l(l+1)\right] \psi(s)=0 .
$$

The indical ideal is given by

$$
J_{t}=\left(\theta_{t}^{2}-\theta_{t}-l(l+1)\right)=(\theta+l)(\theta-(l+1))
$$

From this, we guess that the admissible solution would be similar to $t^{(l+1)}$ around $t=0$, i.e. $\sim(1-s)^{l+1}$ around $s=1$.

Hence we assume one of the solution of the given problem to be $\phi(s)=s^{\sqrt{\alpha}}(1-$ $s)^{(l+1)} f(s)$. Then the differential equation for $f(s)$ is given by

$$
P(s) f^{\prime \prime}(s)+Q(s) f^{\prime}(s)+[-\beta+l(l+1)+(l+1)(2 \sqrt{\alpha}+1)]\left(s^{2}-s\right) f(s)=0,
$$

with polynomials $P(s)$ and $Q(s)$. (The explicit form of them are omitted.) 
We obtain one of possible $f(s)$ when the following is satisfied:

$$
-\beta+l(l+1)+(l+1)(2 \sqrt{\alpha}+1)=0 .
$$

In this case, the differential operator in the left side is subdued to the decomposition:

$$
\left(P(s) \frac{d}{d s}+Q(s)\right) \frac{d}{d s}
$$

The unknown $f$ could be constant, because it could be annihilated by the rightmost component of the decomposition,

$$
\frac{d f(s)}{d s}=0 .
$$

In the same way as the former example, we compute the solution of the lowest eigenenergy $\phi(s)$ and then construct the other solutions $\psi_{n}(s):=\phi(s) y_{n}(s)$. At first we compute and obtain

We also have

$$
\pi(s)=\sigma(s)\left(\frac{\phi^{\prime}(s)}{\phi(s)}\right)=-(l+1+\sqrt{\alpha}) s+\sqrt{\alpha} .
$$

$$
\tau(s)=\tilde{\tau}(s)+2 \pi(s)=(2 l+3+\sqrt{\alpha}) s+2 \sqrt{\alpha}+1 .
$$

The quadratic equation for $\pi$ is simplified to

$$
\pi^{2}(s)+\pi(s)\left[\tilde{\tau}(s)-\sigma^{\prime}(s)\right]+\tilde{\sigma}(s)-k \sigma(s)=[\lambda-\beta+l(l+1)+(l+1)(1+\sqrt{\alpha})]\left(s^{2}-s\right)=0 .
$$

Hence it requires the relation

$$
\lambda-\beta+l(l+1)+(l+1)(1+2 \sqrt{\alpha})=0,
$$

wherein $\lambda$ is equal to $\lambda_{n}$. We have

$$
\begin{aligned}
\lambda_{n} & =-n \frac{d}{d s}[\tilde{\tau}(s)+2 \pi(s)]-n(n-1) \sigma^{\prime \prime}(s) / 2 \\
& =n[n+2 \sqrt{\alpha}+2 l+2] \\
& =\lambda=\beta-l(l+1)-(l+1)(1+2 \sqrt{\alpha}) .
\end{aligned}
$$

From this equation, for each $n$, we obtain $\alpha_{n}$

$$
\alpha_{n}=\frac{1}{4}\left(\frac{\beta}{n+l+1}-(n+l+1)\right)^{2} .
$$

As for the weight function $\rho(s)$ and the relevant polynomials $y_{n}$ of Eq. 114, we have

$$
\rho(s)=s^{2 \sqrt{\alpha}}(1-s)^{2 l+1}
$$

and

$$
y_{n}(s)=B_{n} s^{-2 \sqrt{\alpha}}(1-s)^{-(2 l+1)} \frac{d^{n}}{d s^{n}}\left[s^{n+\sqrt{\alpha}}(1-s)^{n+2 l+1}\right] .
$$

Hence we get the solutions to the given problem by $\psi_{n}(s)=\phi(s) y_{n}(s)$.

\section{Summary and Discussion}

In this part of the article, we review basic ideas of the theory of D-modules, and we see how they would be applied to quantum mechanics. Furthermore, to solve the eigenvalue problem of the Schrödinger equation, we present an alternative algorithm for that of NikiforovUvarov, using a bit of idea of the theory D-modules. In the application of this algorithm in the one-dimensional Schrödinger equation, we extract the approximate form of the solution 
$A(s)$ around the singularities, by using the indical ideal and its decomposition. Then we write the lowest-eigenvalue solution $\phi(s)$ as the product of that approximation $A(s)$ and a enveloping function $f(s)$, by $\phi(s)=A(s) f(s)$. This representation allows us to decompose the differential operator in the differential equation for $f(s)$ without much trouble, and we could get an admissible solution of $f(s)$ from the decomposed component of the operator. (In this part lies the essential difference from the Nikiforov-Uvarov method, which computes $\phi(s)$ after the preparation of a linear polynomial $\pi(s)$. In contrast, we compute $\pi(s)$ from $\phi(s)$.) Once we have obtained $\phi(s)$, we can construct a formula to compute the eigenvalue. We assume the general solutions of the Scrödinger equation of the form $R_{n}(s)=\phi(s) y_{n}(s)$ with relevant polynomials $y_{n}(s)$; and we compute $y_{n}(s)$, as well as the corresponding eigenvalue, in the framework of the theory of hypergeometric differential equation.

From the viewpoint of algebraic geometry, we can interpret the procedure in the following way; at first, we cull out the possibly singular part of the solution; then we resolute the singularity; then the differential operator becomes much more tamed, from which we obtain a physically admissible non-singular solution. The computability of exact solutions is guaranteed only because of the simplicity of the problem: as the one-dimensional time-independent Schrödinger equation is "holonomic", the dimension of its solution set is rigorously limited by Cauchy-Kowalevskii-Kashiwara theorem. However, in higher dimensions, or even in the time-dependent one-dimensional case, the operator is not holonomic; the solutions are set free to broad diversity beyond the limitation which the theorem compels in the holonomic case. Owing to this versatility in the higher dimension, therefore, it seems that it is almost impossible to find exact solutions; instead, we would resort to approximation; we would be obliged to change the methodology, possibly from the non-commutative operators to the polynomials in commutative algebra, for the latter is the most practical way which the present circumstance permits us to employ, even if it is far from being panacea. It shall be an endeavor in quantum mechanics to establish the tactics to solve general and actual problems in three-dimensional space - such as chemistry- by the rigid methods of algebra.

\section{References}

[1] A Kikuchi and I Kikuchi. Computational algebraic geometry and quantum mechanics: An initiative toward post contemporary quantum chemistry. Journal of Multidisciplinary Research and Reviews, 1:47-79, 2019.

[2] Cüneyt Berkdemir. Application of the nikiforov-uvarov method in quantum mechanics. Theoretical Concepts of Quantum Mechanics, page 225, 2012.

[3] H Karayer, D Demirhan, and F Büyükkılıç. Extension of nikiforov-uvarov method for the solution of heun equation. Journal of Mathematical Physics, 56(6):063504, 2015.

[4] B Gönül and K Köksal. A search on the nikiforov-uvarov formalism. Physica Scripta, 75(5):686, 2007.

[5] Arnold F Nikiforov and Vasilii Borisovich Uvarov. Special functions of mathematical physics, volume 205. Springer, 1988.

[6] W Robin. On the rodrigues formula solution of the hypergeometric-type differential equation. In International Mathematical Forum, volume 8, pages 1455-1466, 2013.

[7] Wolfram Decker, Gert-Martin Greuel, Gerhard Pfister, and Hans Schönemann. SINGULAR 4-1-2 - A computer algebra system for polynomial computations. http: //www.singular.uni-kl.de, 2019.

[8] Jason Bell, Albert Heinle, and Viktor Levandovskyy. On noncommutative finite factorization domains. Transactions of the American Mathematical Society, 369(4):26752695, 2017.

[9] Mark Giesbrecht, Albert Heinle, and Viktor Levandovskyy. Factoring differential operators in n variables. arXiv preprint arXiv:1404.0002, 2014. 
[10] A. Kikuchi. An approach to first principles electronic structure computation by symbolic-numeric computation. QScience Connect, 2013:14, 2013. http://dx.doi.org/10.5339/connect.2013.14.

[11] Anna-Laura Sattelberger and Bernd Sturmfels. D-modules and holonomic functions. arXiv preprint arXiv:1910.01395, 2019.

[12] Jan-Erik Björk. Analytic D-modules and applications, volume 247. Springer Science \& Business Media, 2013. 